\title{
Role of the presence of transition-metal atoms at the antisites in CrAs, CrSe and VAs zinc-blende compounds
}

\author{
K Özdog̃an†, I Galanakisł, B Aktaş†, and E Şaşığlu§ \\ † Department of Physics, Gebze Institute of Technology, Gebze, 41400, Kocaeli, \\ Turkey \\ $\ddagger$ Department of Materials Science, School of Natural Sciences, University of \\ Patras, GR-26504 Patra, Greece \\ $\S$ Institut für Festkörperforschung, Forschungszentrum Jülich, D-52425 Jülich, \\ Germany and Fatih University, Physics Department, 34500, Büyükçekmece, \\ İstanbul, Turkey \\ E-mail: \\ kozdogan@gyte.edu.tr, galanakis@upatras.gr,e.sasioglu@fz-juelich.de
}

\begin{abstract}
In a recent publication [Galanakis I et al 2006 Phys. Rev. B 74 $140408(\mathrm{R})]$ we have shown that in the case of CrAs and related transition-metal chalcogenides and pnictides, crystallizing in the zinc-blende structure, the excess of the transition-metal atoms leads to half-metallic ferrimagnetism. The latter property is crucial for spintronic applications with respect to ferromagnets due to the lower stray fields created by these materials. We extend this study to cover the case where the transition-metal atoms sitting at antisites are not identical to the ones in the perfect sites. In Cr-based compounds, the creation of Mn antisites keeps the half-metallic ferrimagnetic character produced also by the $\mathrm{Cr}$ antisites. In the case of VAs, $\mathrm{Cr}$ and $\mathrm{Mn}$ antisites keep the half-metallic character of VAs (contrary to $\mathrm{V}$ antisites) due to the larger exchange-splitting exhibited by these atoms.

PACS numbers: 75.47.Np, 75.50.Cc, 75.30.Et
\end{abstract}

\section{Introduction}

The rapid emergence of the field of spintronics (also known as magnetoelectronics [1]) brought to the center of scientific research the so-called half-metallic ferromagnets (like Heusler alloys [2, 3, 7, or some oxides 44). These compounds present metallic behavior for one spin-band while they are semiconducting or insulators for the other spin-band, resulting to perfect spin-polarization, at least for the bulk, at the Fermi level. For realistic application ferromagnets create large stray fields and thus lead to considerable undesirable energy losses. Thus, to this respect the case of halfmetallic ferrimagnets is more interesting. There are several ways to create a halfmetallic ferrimagnet, either by doping a semiconductor like FeVSb [5] or due to the simultaneous presence of $\mathrm{Mn}$ and another transition-metal atom in the case of alloys with small total moment due to the small number of valence electrons (e.g. FeMnSb 
[3] or $\mathrm{Mn}_{2} \mathrm{VAl}$ [7, 6]). Recently Akai and Ogura have proposed another route to fullycompensated half-metallic ferrimagnetism based on the doping of diluted magnetic semiconductors 8 .

Except Heusler and oxides, also transition-metal chalcogenides like CrAs and pnictides like CrSe are known to present half-metallic ferromagnetism when they crystallize in the metastable zinc-blende structure. The first experimental evidence was provided in the case of CrAs thin-films by the group of Akinaga in 2000 [9] and many more experiments have confirmed these results [10. Experiments agree with prediction of ab-initio calculations performed by several groups [11, 12, 13, 14. In the case of the half-metallic ferromagnets like $\mathrm{CrAs}$ or CrSe, the gap in the minority-spin band arises from the hybridization between the p-states of the $s p$ atom and the tripledegenerated $t_{2 g}$ states of the transition-metal and as a result the total spin-moment, $M_{t}$, follows the Slater-Pauling (SP) behavior being equal in $\mu_{B}$ to $Z_{t}-8$ where $Z_{t}$ the total number of valence electrons in the unit cell [11. Recently theoretical works have appeared attacking also some crucial aspects of these alloys like the exchange bias in ferro-/antiferromagnetic interfaces [15, the stability of the zinc-blende structure [16, the dynamical correlations [17, the interfaces with semiconductors [18, the exchange interaction [19] and the temperature effects [20].

In a recent publication [21, we have investigated the properties of several compounds (CrAs, CrSb, CrSe, CrTe, VAs and MnAs) when we create an excess of the transition metal atoms; transition metal atoms occupy also antisites occupied in the perfect compound by the $s p$ atom. To perform this study we used the fullpotential nonorthogonal local-orbital minimum-basis band structure scheme (FPLO) and we simulated disorder via the coherent potential approximation [22]; we also used the theoretical equilibrium lattice constants for which the perfect compounds are halfmetallic ferromagnets [23]. The most interesting case was the Cr-based compounds. The Cr-impurities in the case of antisites at the sublattice occupied by the $s p$ atoms couple antiferromagnetically to the existing $\mathrm{Cr}$ atoms at the ideal sites and destroy ferromagnetism. But these compounds stay half-metallic for large concentration of antisites exhibiting half-metallic ferrimagnetism. Also in $\mathrm{V}_{1+x} \mathrm{As}_{1-x}$ compound the $\mathrm{V}$ atoms at the antisites couple antiferromagentically to the $\mathrm{V}$ atoms at the perfect sites but the exchange splitting of the $\mathrm{V}$ impurities is small and the Fermi level cross the states of these atoms. In this manuscript we expand this study to cover also the case where at the antisites we find transition metal atoms of different chemical type than the perfect sites. More precisely we studied the case of Mn impurites in $\operatorname{Cr}\left[\mathrm{As}_{1}-x \mathrm{Mn}_{x}\right]$ and $\mathrm{Cr}\left[\mathrm{Se}_{1}-x \mathrm{Mn}_{x}\right]$ and the case of $\mathrm{Cr}$ and $\mathrm{Mn}$ impurities in $\mathrm{V}\left[\mathrm{As}_{1}-x \mathrm{Cr}_{x}\right]$ and $\mathrm{V}\left[\mathrm{As}_{1}-x \mathrm{Mn}_{x}\right]$ compounds. We find that in the case of $\mathrm{Cr}$-compounds the half-metallic ferrimagnetic behavior is again present, and the same is also true for the $\mathrm{V}\left[\mathrm{As}_{1}-x(\mathrm{Cr}\right.$ or $\mathrm{Mn})_{x}$ ] compounds since $\mathrm{Cr}$ and $\mathrm{Mn}$ atoms couple antiferomagnetically to the $\mathrm{V}$ atoms at the perfect site and have a larger exchange splitting than the $\mathrm{V}$ impurities in $\mathrm{V}_{1+x} \mathrm{As}_{1-x}$.

\section{Total magnetic moments}

Before going in details of the density of states (DOS) or atomic spin moments we will present our results on the total spin moments. In figure 1 we have included the different cases under study going up to $\mathrm{x}=0.5$ where half of the $s p$ atoms have been substituted by transition metal atoms. Of course such a case is unlikely to be

stable in the zinc-blende structure but we have gone so far to make physics more 


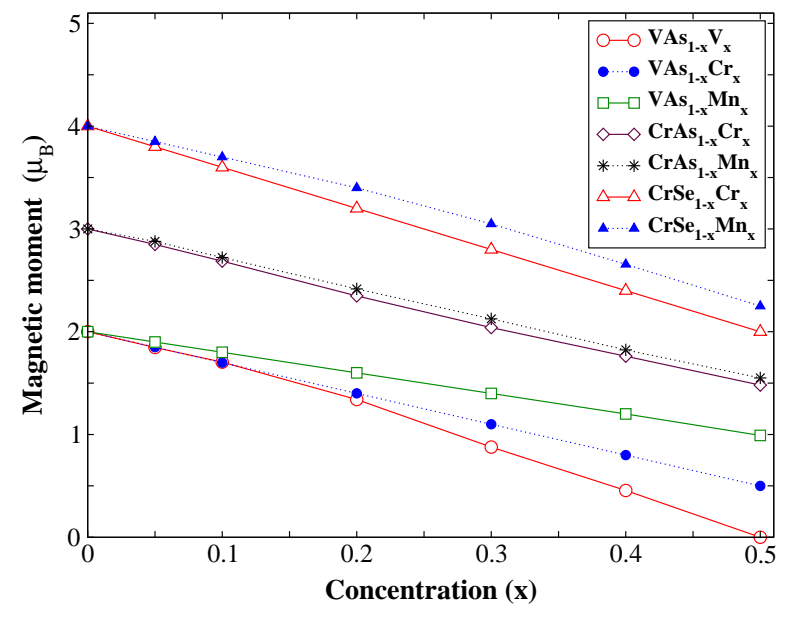

Figure 1. (Color online) Total spin magnetic moment as a function of the concentration $x$ for the studied $\mathrm{T}\left[\mathrm{Z}_{1-x} \mathrm{~T}_{x}\right]$ compounds.

transparent.The perfect CrAs compound has a total spin moment in the unit cell of $3 \mu_{B}$ since there are 11 valence electrons, CrSe with one valence electron more in the unit cell has a spin moment of $4 \mu_{B}$ and VAs with 10 valence electrons a spin moment of $2 \mu_{B}$. In all cases the total spin moment decreases with the concentration, a clear sign of the antiferromagnetic coupling between the transition metal atoms at different sites. In the case of $\mathrm{CrAs}$, substituting $\mathrm{Cr}$ or $\mathrm{Mn}$ for As results to identical behavior (solid black lines with open diamonds and stars, respectively in the figure) and the total spin moment is almost independent of the chemical type of the transition-metal atom at the antisite. This is also true to a lesser extent for CrSe (red open triangles for $\mathrm{Cr}\left[\mathrm{Se}_{1-x} \mathrm{Cr}_{x}\right]$ and blue triangles for $\left.\mathrm{Cr}\left[\mathrm{Se}_{1-x} \mathrm{Mn}_{x}\right]\right)$. For the same concentration $\mathrm{x}$ the compound with Mn has a slightly larger total spin moment and as the concentration increases this difference also increases.

In the case of VAs the differences are more pronounced. When we substitute $\mathrm{V}$ for As, $\mathrm{V}\left[\mathrm{As}_{1-x} \mathrm{~V}_{x}\right]$, the $\mathrm{V}$ atoms at the ideal and the antisites are antiferromagnetically coupled. As the concentration increases the absolute value of the $\mathrm{V}$ spin moments decreases and for $\mathrm{x}=0.5$ the total spin moment is almost zero and the $\mathrm{V}$ atoms are almost non-magnetic. $\mathrm{Cr}$ and $\mathrm{Mn}$ impurities, on the other hand, lead to larger total spin moment and the resulting compounds are ferrimagnets with strong magnetic elements. Overall the $\mathrm{V}\left[\mathrm{As}_{1-x} \mathrm{Mn}_{x}\right]$ compounds have a larger spin moment than the $\mathrm{V}\left[\mathrm{As}_{1-x} \mathrm{Cr}_{x}\right]$ ones. We should note that although the behavior of the total spin moments reveals the existence of ferrimagnetism it does not give any information about the half-metallic character and we should look how the DOS changes with the concentration $x$ for every case to be sure if the half-metallicity is preserved or lost.

\section{The Cr-based compounds}

The first family of compounds under study is the CrAs and CrSe alloys. We extended the study presented in reference 21] to cover also the case when we substitute Mn for As or $\mathrm{Se}$. $\mathrm{CrSb}$ is isoelectronic to $\mathrm{CrAs}$ (same number of valence electrons) and $\mathrm{CrTe}$ 
Table 1. Total and atom-resolved spin magnetic moments for the $\operatorname{Cr}\left[\mathrm{Z}_{1-x} \mathrm{Y}_{x}\right]$ compounds where $\mathrm{Z}=\mathrm{As}$ or Se and $\mathrm{Y}=\mathrm{Cr}$ or $\mathrm{Mn}$. As "imp" we denote the transition-metal atoms which are located at antisite positions. Note that the atomic moments have been scaled to one atom.

\begin{tabular}{l|cccc|cccc}
\hline & \multicolumn{4}{|c|}{$\mathrm{Cr}\left[\mathrm{As}_{1-x} \mathrm{Cr}_{x}\right]$} & \multicolumn{4}{c}{$\mathrm{Cr}\left[\mathrm{As}_{1-x} \mathrm{Mn}_{x}\right]$} \\
$x$ & Total & $\mathrm{Cr}$ & $\mathrm{As}$ & $\mathrm{Cr}-\mathrm{imp}$ & Total & $\mathrm{Cr}$ & $\mathrm{As}$ & $\mathrm{Mn}-\mathrm{imp}$ \\
\hline 0 & 3.00 & 3.41 & -0.41 & - & 3.00 & 3.41 & -0.41 & - \\
0.05 & 2.85 & 3.37 & -0.40 & -2.77 & 2.88 & 3.38 & -0.40 & -2.49 \\
0.1 & 2.69 & 3.33 & -0.40 & -2.81 & 2.72 & 3.33 & -0.39 & -2.57 \\
0.2 & 2.35 & 3.24 & -0.40 & -2.88 & 2.42 & 3.25 & -0.38 & -2.66 \\
0.4 & 1.76 & 3.19 & -0.39 & -3.00 & 1.82 & 3.19 & -0.36 & -2.87 \\
\hline \hline & \multicolumn{4}{|c}{$\mathrm{Cr}\left[\mathrm{Se}_{1-x} \mathrm{Cr}_{x}\right]$} & & \multicolumn{5}{c}{$\mathrm{Cr}\left[\mathrm{Se}_{1-x} \mathrm{Mn}_{x}\right]$} \\
$x$ & Total & $\mathrm{Cr}$ & $\mathrm{Se}$ & $\mathrm{Cr}-\mathrm{imp}$ & Total & $\mathrm{Cr}$ & $\mathrm{Se}$ & $\mathrm{Mn}-\mathrm{imp}$ \\
\hline 0 & 4.00 & 4.20 & -0.20 & - & 4.00 & 4.20 & -0.20 & - \\
0.05 & 3.80 & 4.12 & -0.17 & -3.08 & 3.85 & 4.15 & -0.17 & -2.92 \\
0.1 & 3.60 & 4.06 & -0.17 & -3.08 & 3.70 & 4.14 & -0.16 & -2.92 \\
0.2 & 3.20 & 3.96 & -0.17 & -3.08 & 3.40 & 4.11 & -0.16 & -2.93 \\
0.4 & 2.40 & 3.75 & -0.18 & -3.09 & 2.66 & 3.98 & -0.16 & -3.09 \\
\hline \hline
\end{tabular}

is isoelectronic to CrSe, thus they are expected to present similar behavior and we have not included them in our study. In table 1 we present the total and atom-resolved spin magnetic moments for the $\mathrm{Cr}\left[\mathrm{As}_{1-x} \mathrm{Cr}_{x}\right]$ and $\mathrm{Cr}\left[\mathrm{As}_{1-x} \mathrm{Mn}_{x}\right]$ compounds in the upper panel and the $\mathrm{Cr}\left[\mathrm{Se}_{1-x} \mathrm{Cr}_{x}\right]$ and $\mathrm{Cr}\left[\mathrm{Se}_{1-x} \mathrm{Mn}_{x}\right]$ compounds in the lower panel. In figure 2 we present the atom-resolved DOS for all four cases and for $\mathrm{x}=0.2$.

In $\mathrm{CrAs}$ the Fermi level is close the upper-energy edge of the gap and in $\mathrm{CrSe}$ somewhat deeper in the gap. Thus half-metallicity is easier to be kept for the Secompound. Overall the $\mathrm{Cr}$ atoms at the perfect $\left(\begin{array}{lll}0 & 0 & 0\end{array}\right)$ site show similar DOS for all cases and the spin moment of these $\mathrm{Cr}$ atoms, as can be seen in table 1, present small variations with the impurities concentration at the antisites (the differences are somewhat larger for the Se compounds). Each Cr atom at the perfect site has four As or Se nearest-neighbors. As we substitute $\mathrm{Cr}$ or Mn for As or Se, the immediate environment of the $\mathrm{Cr}$ atoms at the perfect sites changes and instead of hybridizing with the p-states of As or Se now they hybridize with the $t_{2 g}$ states of $\mathrm{Cr}$ or $\mathrm{Mn}$ impurities leading to a small decrease of the spin moment of these $\mathrm{Cr}$ atoms at the ideal sites.

Both $\mathrm{Cr}$ and Mn impurities have all their five majority d-states (spin-down states with respect to the $\mathrm{Cr}$ atoms at the perfect sites) occupied as well as the minority (spin-up) double-degenerated $e_{g}$ states occupied. The spin moment at the impurity is around $-3 \mu_{B}$ being the total number of the uncompensated spins. This is clearly seen in figure 2 where we present with red dashed lines the DOS of the impurity atoms at the antisites. For majority spins (negative values of DOS) there is one large peak occupied containing all five d-states. For the minority spins (positive values of DOS) there is a much smaller peak occupied containing the double-degenerated $e_{g}$ electrons. The $e_{g}$ electrons are well localized since they do not hybridize due to symmetry reasons neither with the $t_{2 g}$ electrons of the transition metal atoms nor with the $p$ electrons of As or Se and thus they are occupied for both spin directions. On the other hand the triple-degenerated $t_{2 g}$ states present strong hybridization with their environment and a considerable large exchange splitting. As a result the majority $t_{2 g}$ states are completely occupied and the minority $t_{2 g}$ states are empty leading to the spin moment of around $-3 \mu_{B}$. Mn presents a larger exchange-splitting than $\mathrm{Cr}$ and thus also in its 


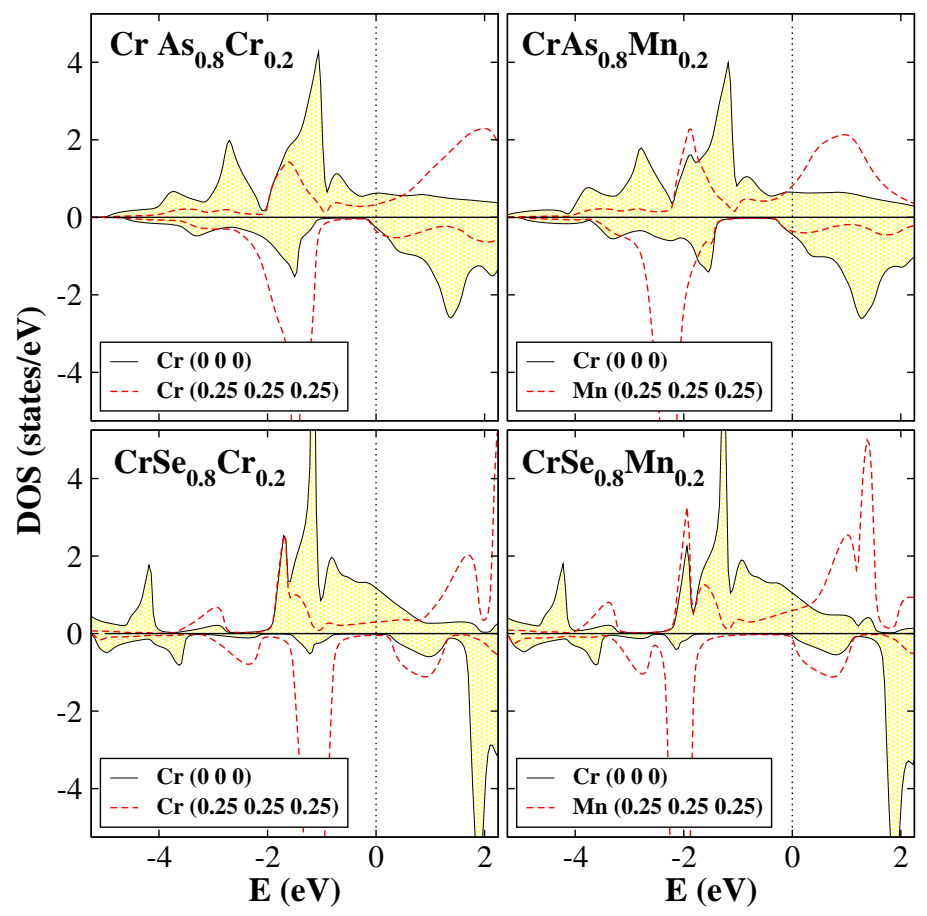

Figure 2. (Color online) Atom-resolved DOS for the $\mathrm{Cr}$ atom at the ideal (0 $00)$ site and for the transition-metal impurity at the $\left(\begin{array}{lll}1 & \frac{1}{4} & \left.\frac{1}{4}\right)\end{array}\right)$ antisite for the $\mathrm{Cr}\left[\mathrm{Z}_{0.8} \mathrm{Y}_{0.2}\right]$ compounds where $\mathrm{Z}=\mathrm{As}$ or $\mathrm{Se}$ and $\mathrm{Y}=\mathrm{Cr}$ or Mn. Positive values of DOS correspond to spin-up electrons and negative values to spin-down electrons. The energy scale is such that the zero energy corresponds to the energy of the Fermi level. Notice that the majority electrons correspond to spin-up electrons for the $\mathrm{Ct}$ at the ideal sites and to spin-down electrons for the $\mathrm{Cr}$ or $\mathrm{Mn}$ impurities at the antisites. The vice versa is true for the minority-spin electrons.

case the spin-moment can not surpass the value of -3 . The small deviations from this ideal value in table 1 come from the hybridization of the $\mathrm{Cr}$ or $\mathrm{Mn}$ impurities with the d-states of the $\mathrm{Cr}$ atoms at the perfect sites.

As we have already mentioned the Cr atoms at the ideal $\left(\begin{array}{lll}0 & 0 & 0\end{array}\right)$ sites do not change their DOS with the concentration of the $\mathrm{Cr}$ or Mn impurities and they do not affect the gap (neither its location or its width). Also $\mathrm{Mn}$ and $\mathrm{Cr}$ impurities present a large exchange splitting and there is a considerable gap between the occupied majority (spin-down) d-states and the unoccupied antibonding states. The width of this gap is much larger for the $\mathrm{Mn}$ impurities (this is clear if we look at the CrSe case) where the exchange splitting between the majority (spin-down) occupied d-states and the minority (spin-up) unoccupied $t_{2 g}$-states is larger than for the $\mathrm{Cr}$ atoms. All these phenomena result in the persistence of the gap and thus of the half-metallicity upon doping with either $\mathrm{Cr}$ or $\mathrm{Mn}$ for As or Se. Also as we mentioned already the impurities are antiferromagentically coupled to the $\mathrm{Cr}$ atoms at the ideal sites leading to the desirable half-metallic ferrimagnetism.

Before proceeding to the V-based compounds we have to shortly also discuss the behavior of the sp atoms. Both As and Se have their minority p-states completely 


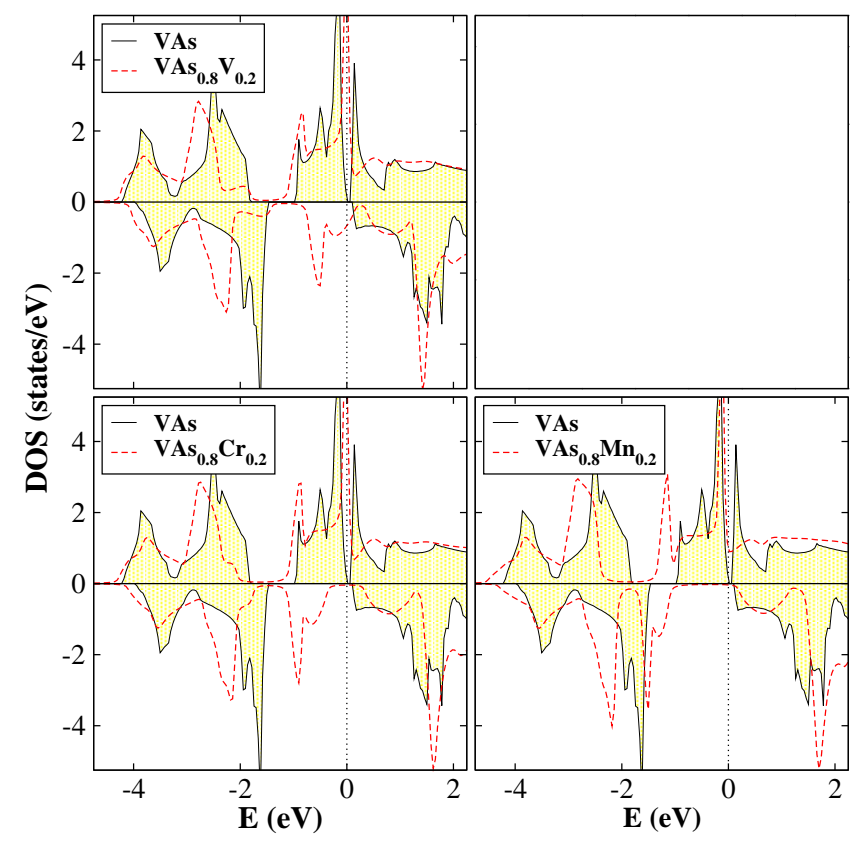

Figure 3. (Color online) Total DOS for the $\mathrm{V}\left[\mathrm{As}_{0.8} \mathrm{~V}_{0.2}\right]$ (upper panel), $\mathrm{V}\left[\mathrm{As}_{0.8} \mathrm{Cr}_{0.2}\right]$ (lower left panel) and $\mathrm{V}\left[\mathrm{As}_{0.8} \mathrm{Mn}_{0.2}\right]$ (lower right panel) compounds with respect to the perfect VAs (shaded area).

occupied while for the majority p-states some of the weight is over the Fermi level leading to small negative spin moments as seen in table 1. The origin of the antiferrimagnetic coupling between the transition-metal atom and the sp-atom is discussed in detail in reference [12. Thus the change in the concentration of As or Se only slightly affects the properties of these atoms and the small change in their spin moments arises mainly due to the change in the spin moments of the $\mathrm{Cr}$ atoms at the ideal site through the hybridization between the $\mathrm{Cr} d$ states and the As or Se p states.

\section{The V-based compounds}

The second case under study is the presence of $\mathrm{Cr}$ and $\mathrm{Mn}$ impurities in VAs. In reference [21, we have shown that the excess of $\mathrm{V}, \mathrm{V}\left[\mathrm{As}_{1-x} \mathrm{~V}_{x}\right]$ family, destroys the half-metallic character of the ideal VAs alloy. In figure 3 we have gathered the total DOS (red dashed line) for the $\mathrm{V}\left[\mathrm{As}_{0.8} \mathrm{Y}_{0} .2\right]$ with $\mathrm{Y}=\mathrm{V}, \mathrm{Cr}$ or Mn compared to the total DOS for the ideal VAs alloy (shaded area). It is obvious that $\mathrm{Cr}$ and $\mathrm{Mn}$ impurities due to the larger exchange splitting, which they present with respect to the $\mathrm{V}$ impurities, keep the half-metallic character although especially for $\mathrm{Cr}$ the width of the gap is severely reduced; for Mn the occupied spin-down states are pushed very low in energy and the width of the gap is almost unchanged. We will discuss in detail this phenomenon in the following paragraph. For now let's concentrate on the behavior around the Fermi level of the majority DOS. In perfect VAs the Fermi level falls within a very sallow majority gap so that only the majority $e_{g}$ states are occupied leading to 


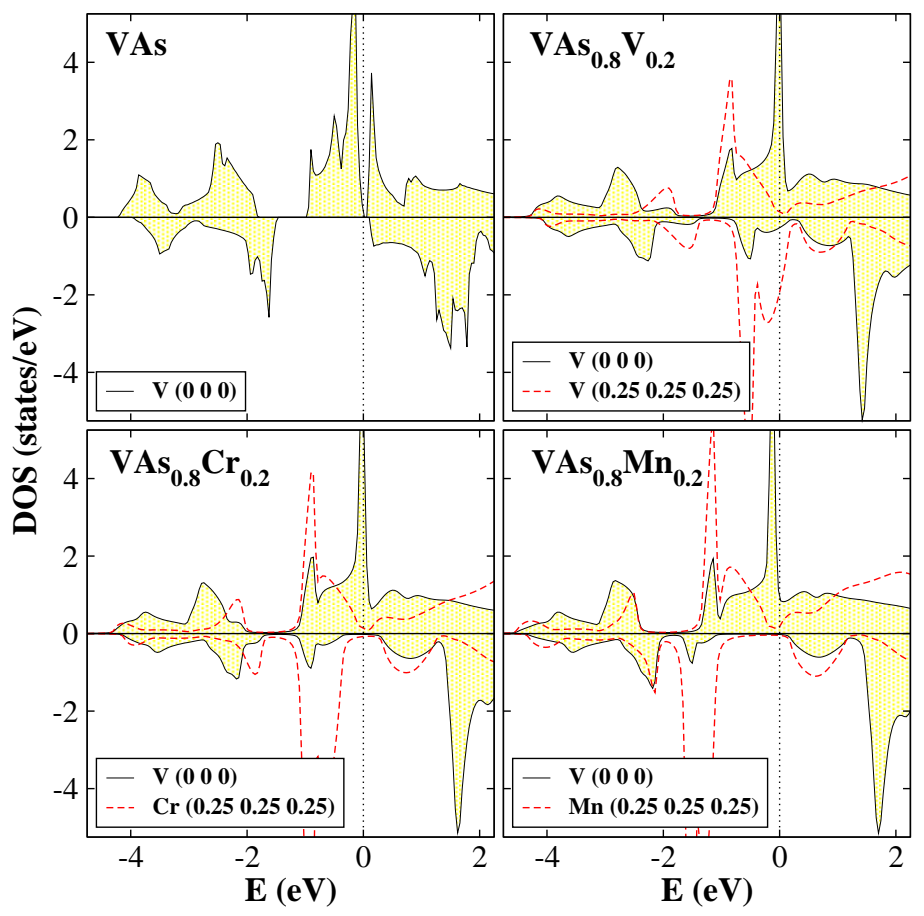

Figure 4. (Color online) Atom-resolved DOS for the $\mathrm{V}$ atom at the ideal (0 0 0) site and for the transition-metal impurity at the $\left(\frac{1}{4} \frac{1}{4} \frac{1}{4}\right)$ antisite for the $\mathrm{V}\left[\mathrm{As}_{0.8} \mathrm{Y}_{0.2}\right]$ compounds where $\mathrm{Y}=\mathrm{V}, \mathrm{Cr}$ or $\mathrm{Mn}$.

a total spin moment of exactly $2 \mu_{B}$ [12. When we substitute $\mathrm{V}, \mathrm{Cr}$ or $\mathrm{Mn}$ for As, even at low concentration of the transition-metal impurities, the large majority peak of $\mathrm{V}$ just below the Fermi level moves slightly higher in energy and now the Fermi level falls within this peak. In figure 4 where we present the atom-resolved DOS, it is clear that this peak comes exclusively from the $\mathrm{V}$ atoms at the ideal $\left(\begin{array}{lll}0 & 0 & 0\end{array}\right)$ sites and is irrelevant to the impurities.

As mentioned above in figure 4 we have drawn the atom-resolved DOS for the transition metal atoms in the case of the perfect VAs and the $\mathrm{V}\left[\mathrm{As}_{0.8} \mathrm{Y}_{0} .2\right]$ with $\mathrm{Y}=$ $\mathrm{V}, \mathrm{Cr}$ or Mn alloys(V atoms at the ideal $\left(\begin{array}{lll}0 & 0 & 0\end{array}\right)$ sites with the shaded region and $\mathrm{V}$, $\mathrm{Cr}$ or $\mathrm{Mn}$ impurities at the $(0.250 .250 .25)$ antisites with the dashed red lines). In table 2 we have gathered the total and spin magnetic moments for the $\mathrm{V}\left[\mathrm{As}_{1-x} \mathrm{Cr}_{x}\right]$ and $\mathrm{V}\left[\mathrm{As}_{1-x} \mathrm{Mn}_{x}\right]$ compounds as a function of the concentration of the impurities. We will start our discussion from the $\mathrm{V}$ atoms at the ideal sites. Due to the change in their environment $e_{g}$ and $t_{2 g}$ majority states are not any more separated by a tiny gap but they slightly overlap so that that the Fermi level is not in a majority tiny gap any more. Comparing the values for $\mathrm{V}\left[\mathrm{As}_{1-x} \mathrm{~V}_{x}\right]$ in table 1 of reference [21] and the values for $\mathrm{V}\left[\mathrm{As}_{1-x} \mathrm{Cr}_{x}\right]$ and $\mathrm{V}\left[\mathrm{As}_{1-x} \mathrm{Mn}_{x}\right]$ in table 2 of the present manuscript, we can identify a global trend in the behavior of the $\mathrm{V}$ at the perfect site. As we move from the heavier element $\mathrm{Mn}$ to the lighter $\mathrm{V}$, the increase of the impurities concentration pushes the Fermi level deeper in the large peak located around the Fermi level resulting to smaller V spin magnetic moments. To make it more clear we consider the case for 
Effect of antisites in CrAs and related compounds

Table 2. Total and atom-resolved spin magnetic moments for the $\mathrm{V}\left[\mathrm{As}_{1-x} \mathrm{Y}_{x}\right]$ compounds where $\mathrm{Y}=\mathrm{Cr}$ or $\mathrm{Mn}$. As "imp" we denote the transition-metal atoms which are located at antisite positions. Note that the atomic moments have been scaled to one atom.

\begin{tabular}{l|cccc|cccc}
\hline & \multicolumn{4}{|c|}{${\mathrm{V}\left[\mathrm{As}_{1-x} \mathrm{Cr}_{x}\right]}$} & \multicolumn{4}{c}{$\mathrm{V}\left[\mathrm{As}_{1-x} \mathrm{Mn}_{x}\right]$} \\
$x$ & Total & $\mathrm{V}$ & As & Cr-imp & Total & $\mathrm{V}$ & As & Mn-imp \\
\hline 0 & 2.00 & 2.27 & -0.27 & - & 2.00 & 2.27 & -0.27 & - \\
0.05 & 1.85 & 2.19 & -0.25 & -2.07 & 1.90 & 2.25 & -0.26 & -2.02 \\
0.1 & 1.70 & 2.13 & -0.25 & -2.05 & 1.80 & 2.23 & -0.25 & -2.03 \\
0.2 & 1.40 & 1.98 & -0.25 & -1.99 & 1.60 & 2.21 & -0.25 & -2.05 \\
0.3 & 1.10 & 1.82 & -0.21 & -1.91 & 1.40 & 2.19 & -0.25 & -2.07 \\
0.4 & 0.80 & 1.66 & -0.19 & -1.86 & 1.20 & 2.18 & -0.24 & -2.10 \\
0.5 & 0.50 & 1.56 & -0.18 & -1.93 & 0.99 & 2.19 & -0.24 & -2.15 \\
\hline \hline
\end{tabular}

$\mathrm{x}=0.2$. In the case of $\mathrm{V}$ impurities the $\mathrm{V}$ at the ideal site has a spin moment of 1.7 $\mu_{B}$, for $\mathrm{Cr}$ impurities its spin moment is $1.98 \mu_{B}$ while for $\mathrm{V}\left[\mathrm{As}_{0.8} \mathrm{Mn}_{0.2}\right]$ the spin moment of the $\mathrm{V}$ at the ideal site is $2.21 \mu_{B}$ very close to the value for the perfect VAs compound of $2.27 \mu_{B}$. Due to this larger drop in the spin moment of the $\mathrm{V}$ atoms at the perfect site for the lighter element, we observe the behavior of the total spin moment in figure 1. For $\mathrm{V}$ impurities the total spin moment is almost zero (in reality slightly negative) for $\mathrm{x}=0.5$ and for the case of $\mathrm{Cr}$ impurities the total spin moment is $0.5 \mu_{B}$ for $\mathrm{z}=0.5$. On the other hand for $\mathrm{Mn}$ impurities the spin moment of $\mathrm{V}$ atoms remains almost constant as we can see in table 2 and the drop in the total moment comes exclusively from the increase in the concentration of Mn being $1 \mu_{B}$ for $\mathrm{x}=0.5$ double the one for the $\mathrm{Cr}$ impurities case.

We still have not discussed the behavior of the impurity atoms in the case of VAs. From the table 2 it is obvious that, contrary to Cr-based compounds, the impurity $\mathrm{Cr}$ or $\mathrm{Mn}$ atoms have a spin magnetic moment of around $-2 \mu_{B}$, while the $\mathrm{V}$ impurity atoms (see table 1 in reference 21]) have a spin moment of around $1 \mu_{B}$. To understand this behavior we have to look at the atom-resolved DOS in figure 4. The exchange splitting of the $\mathrm{V}$ impurities at the antisites is very small and the majority (spin-down) and minority (spin-up) double degenerated $e_{g}$-states are completely occupied (peaks with the higher intensity located around $-1 \mathrm{eV}$ ). Also one of the minority and two of the majority triple-degenerated $t_{2 g}$ states are occupied resulting in a spin moment of around $-1 \mu_{B}$ and the Fermi level is crossing the spindown band of the $t_{2 g}$ states. For $\mathrm{Cr}$ and $\mathrm{Mn}$ impurities the exchange splitting is larger and all three majority (spin-down) $t_{2 g}$-states are occupied while the picture for the minority states does not change and consecutively the spin moment of the impurity is around $-2 \mu_{B}$. Also this results to a spin-down gap separating the fully occupied d-states from the antibonding states lying higher in energy and thus to half-metallic ferrimagnetism. The exchange splitting is larger for $\mathrm{Mn}$ than $\mathrm{Cr}$ and the spin-down gap is larger in the former case. As we change the concentration of the impurity atoms their spin moment only slightly changes due to the stronger hybridization with the $\mathrm{V}$ atoms at the ideal sites. Finally we should note that as we decrease the number of valence electrons and pass from CrSe to CrAs and then to VAs the ionicity of the compounds becomes smaller and so do the gaps created by the impurity atoms (smaller exchanged splitting for the same impurity atom when it is found in environment with smaller ionicity). 


\section{Conclusion}

We have complemented our study presented in a recent publication [Galanakis I et al 2006 Phys. Rev B $\mathbf{7 4}$ 140408(R)] where we have shown that in the case of $\mathrm{CrAs}$ and related transition-metal chalcogenides and pnictides, crystallizing in the zinc-blende structure, the excess of the transition-metal atoms leads to half-metallic ferrimagnetism. In this contribution we show that there may be additional advantages when the excess of the transition-metal atom is not of the same chemical type as the one at the perfect lattice sites. In CrAs and CrSe, the creation of Mn antisites keeps the half-metallic character and the $\mathrm{Mn}$ impurities are antiferromagnetically coupled to the $\mathrm{Cr}$ ones leading to half-metallic ferrimagnetism. The larger exchange splitting of the $\mathrm{Mn}$ atoms with respect to the $\mathrm{Cr}$ ones makes the half-metallic ferrimagnetism even more robust since the gap-width is larger with respect to the case of $\mathrm{Cr}$ antisites. Even in VAs, which looses its half-metallic character upon creation of $\mathrm{V}$ antisites, the appearance of $\mathrm{Cr}$ or $\mathrm{Mn}$ antisites is accompanied with the appearance of half-metallic ferrimagnetism. These results may suggest a new way to achieve stable half-metallic ferrimagnets, which is crucial for spintronic applications with respect to half-metallic ferromagnets due to the lower stray fields created by these materials.

\section{Acknowledgments}

Authors acknowledge the computer support of the "Leibniz Institute for Solid State and Materials Research Dresden".

\section{References}

[1] Žutić I, Fabian J and Das Sarma S 2004 Rev. Mod. Phys. 76323

[2] de Groot R A, Mueller F M, van Engen P G and Buschow K H J 1983 Phys. Rev. Lett. 50 2024

[3] Galanakis I, Dederichs P H and Papanikolaou N 2002 Phys. Rev. B 66134428

[4] Soulen Jr R J, Byers J M, Osofsky M S, Nadgorny B, Ambrose T, Cheng S F, Broussard P R, Tanaka C T, Nowak J, Moodera J S, Barry Q and Coey J M D 1998 Science 282 85; Park J H, Vescovo E, Kim H J, Kwon C, Ramesh R and Venkatesan T 1998 Nature 392 794; Parker J S, Watts S M, Ivanov P G and Xiong P 2002 Phys. Rev. Lett. 88 196601; Kämper K P, Schmitt W and Güntherodt G 1987 Phys. Rev. Lett. 592788

[5] van Leuken H and de Groot R A 1995 Phys. Rev. Lett. 741171

[6] Özdog̃an K, Galanakis I, Şaşıg̃lu E and Aktaş B 2006 J. Phys.: Condens. Matter 182905 ; Şaşıõlu E, Sandratskii L M and Bruno P 2005 J. Phys.: Condens. Matter 17995

[7] Galanakis I, Dederichs P H and Papanikolaou N 2002 Phys. Rev. B 66 174429; Weht R and Pickett W E 1999 Phys. Rev. 6013006

[8] Akai H and Ogura M 2006 Phys. Rev. Lett. 97026401

[9] Akinaga H, Manago T and Shirai M 2000 Jpn. J. Appl. Phys. 39 L1118.

[10] Deng J J, Zhao J H, Bi J F, Niu Z C, Yang F H, Wu X G, and Zheng H Z 2006 J. Appl. Phys. 99093902 Zhao J H, Matsukura F, Takamura K, Chiba D, Ohno Y, Ohtani K, and Ohno H 2003 Mat. Sci. Semicond. Proc. 6507

[11] Galanakis I and Mavropoulos P 2003 Phys. Rev. B 67104417

[12] Galanakis I 2002 Phys. Rev. B 66012406

[13] Zhao Y J and Zunger A 2005 Phys. Rev. B 71 13240; Miao M S and Lambrecht W R L 2005 Phys. Rev. B 71 064407; Shi L J and Liu B G 2005 J. Phys.: Condens. Matter 171209

[14] Shirai M 2004 J. Phys.: Condens. Matter 16 S5525; Shirai M, Seike M, Sato K and KatayamaYoshida H 2004 J. Magn. Magn. Mater. 272-276 344

[15] Nakamura K, Kato Y, Akiyama T, Ito T, and Freeman A J 2006 Phys. Rev. Lett. 96047206

[16] Xie W H, Xu Y Q, Liu B G, and Pettifor D G 2003 Phys. Rev. Lett. 91 037204; idem Phys. Rev. Lett. 91219901 (erratum) 
[17] Chioncel L, Mavropoulos P, Ležaić M, Blügel S, Arrigoni E, Katsnelson M I, and Lichtenstein A I 2006 Phys. Rev. Lett. 96197203

[18] Mavropoulos P, Galanakis I, and Dederichs P H 2004 J. Phys.: Condens. Matter 16 4261; Fong C Y, Qian M C, Pask J E, Yang L H, and Dag S 2004 Appl. Phys. Lett. 84239

[19] Sasioglu E, Galanakis I, Sandratskii L M, and Bruno P 2005 J. Phys.: Condens. Matter 17 3915

[20] Ležaić M, Mavropoulos P, Enkovaara J, Bihlmayer G, and Blügel S 2006 Phys. Rev. Lett. 97 026404; Chioncel L, Katsnelson M I, de Wijs G A, de Groot R A, and Lichtenstein A I 2005 Phys. Rev. B 71 085111; Skomski R and Dowben PA 2002 Europhys. Lett. 58544

[21] Galanakis I, Özdog̃an K, Şaşıõlu E and Aktaş B 2006 Phys. Rev. B 74 140408(R)

[22] Koepernik K and Eschrig H 1999 Phys. Rev. 59 1743; Koepernik K, Velicky B, Hayn R and Eschrig H 1998 Phys. Rev. B 586944

[23] CrAs $(0.565 \mathrm{~nm})$, CrSe $(0.582 \mathrm{~nm})$ and VAs $(0.565 \mathrm{~nm})$. 